\title{
Glücksspiel im europäischen Recht
}

\author{
Wolfgang Heusel
}

Online publiziert: 5. Februar 2010

(C) ERA 2010

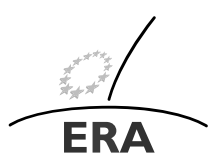

EUROPÄISCHE RECHTSAKADEMIE ACADEMY OF EUROPEAN LAW ACADEMIE DE DROIT EUROPEEN ACCADEMIA DI DIRITTO EUROPEO

\section{Vorbemerkung}

Wie fast schon traditionell für die letzte Ausgabe des Jahres bringt das vorliegende Heft 4/2009 des ERA Forum Beiträge zu unterschiedlichen aktuellen Themen, die Gegenstand von Vorträgen und Debatten auf ERA-Veranstaltungen des vergangenen Jahres waren. Dabei setzen die ersten vier Beiträge zum Glücksspielrecht in Europa einen besonderen thematischen Schwerpunkt, mit dem sich auch dieses Editorial im wesentlichen befasst. Dem stehen weitere Beiträge zu so unterschiedlichen Gebieten wie dem europäischen Umweltrecht, dem europäischen Mediationsrecht oder dem Urteil des Bundesverfassungsgerichts zur Vereinbarkeit des Lissabonner Vertrags mit deutschem Verfassungsrecht gegenüber.

Zunächst setzt sich der Beitrag des Vorsitzenden des Kuratoriums der ERA und Richters am englischen Technology and Construction Court John Toulmin mit Fragen der grenzüberschreitenden Mediation in Verbraucher-, Handels- oder Familiensachen vor nationalen Zivilgerichten insbesondere im Hinblick auf die Mediations-Richtlinie 2008/52 auseinander, die zuvor ausführlich im Rahmen einer ERA-Tagung behandelt worden waren. - Im Bereich des europäischen Wasserrechts arbeitet die ERA seit Jahren mit dem Trierer Institut für deutsches und europäisches Wasserwirtschaftsrecht am Tagungsstandort Brüssel zusammen; der jüngsten Tagung zu aktuellen wasserrechtlichen Fragen entstammen die Beiträge von William Howarth zur Kostenerstattung nach dem Verursacherprinzip im Rahmen der Wasserrahmenrichtlinie und von Fabrice Bin mit einer rechtsvergleichenden Betrachtung zur Verhaltenssteuerung

\section{W. Heusel ( $₫)$}

Europäische Rechtsakademie, Metzer Allee 4, 54295 Trier, Germany

e-mail: wheusel@era.int 
der Wassernutzer durch Abgaben. Von einem weiteren Feld des europäischen Umweltrechts, aus einer Veranstaltung zum dreißigjährigen Bestehen der VogelschutzRichtlinie, kommt der Artikel von Francis Haumont, der sich mit Ausgleichsmaßnahmen für Natura 2000-Gebiete nach Artikel 6 der Habitat-Richtlinie befasst. Schließlich bietet der Beitrag von Malte Beyer-Katzenberger eine englischsprachige Darstellung des Lissabon-Urteils des Bundesverfassungsgerichts vom 30. Juni 2009 und eine kritische Auseinandersetzung mit einigen der Schlussfolgerungen des Gerichts.

\section{Die Regulierung des europäischen Glücksspielmarktes}

Lotterien und Glücksspiele bilden seit jeher eine zuverlässige staatliche Einnahmequelle, aus der zahlreiche mehr oder weniger gemeinnützige ,good causes“ wie der Breiten- und Spitzensport, die Pferdezucht oder kulturelle Projekte finanziert werden. Auch wenn eine sekundärrechtliche Regulierung dieses wichtigen europäischen Wirtschaftssektors weiterhin aussteht, ist die Veranstaltung von Glücksspielen nach ständiger Rechtsprechung des Europäischen Gerichtshofs als Dienstleistung und damit als Wahrnehmung einer der vier Grundfreiheiten des Binnenmarkts anerkannt. Einschränkungen lassen sich daher nur durch zwingende Erfordernisse des Gemeinwohls im Rahmen des Erforderlichen und Verhältnismäßigen (z. B. zur wirksamen Bekämpfung von Spielsucht oder Kriminalität oder aus Gründen des Verbraucherschutzes), in keinem Fall jedoch durch fiskalische Interessen etwa zur Erzielung von Einnahmen für gemeinnützige Zwecke rechtfertigen.

Auf nationaler Ebene sind die Glücksspielmärkte in der EU sehr unterschiedlich ausgestaltet. Die Bandbreite reicht von der völligen Marktabschottung über zahlreiche Zwischenstufen (geschlossene Märkte mit lizenzierten Monopolinhabern, liberalisierte Märkte mit einer begrenzten Zahl bereitgestellter Lizenzen, etc.) bis zu Modellen des offenen Marktzugangs mit oder ohne Lizenzierung. Dabei ist das niemals offen eingestandene, aber in allen Argumentationsmustern immer wieder durchscheinende sehr verständliche Hauptmotiv der Marktabschottung bei den Monopolstaaten die Furcht vor Einnahmeverlusten und dem Risiko der Belastung der allgemeinen Haushalte mit der Finanzierung der ,good causes“, denn faktisch steuerfrei wirtschaftende Internet-Anbieter aus Gibraltar können höhere Gewinnquoten anbieten als staatliche Monopolanbieter mit hoher Abgabenlast.

Es fragt sich aber, ob eine Rechtssicherheit schaffende europäische Rahmenregulierung des Glücksspielmarktes unter Eröffnung adäquater Gewinnabschöpfungsmaßnahmen im Bestimmungsland nicht besser geeignet sein würde, den Schutz sowohl der geltend gemachten Gemeinwohlinteressen als auch der durchaus legitimen, aber nicht grundfreiheitsfesten fiskalischen Interessen der Mitgliedstaaten zu gewährleisten als der bestehende Zustand der Rechtszersplitterung. Zwar hat die Diskussion um die Verabschiedung der Dienstleistungsrichtlinie 2006 noch einmal die geringen Chancen einer europäischen Regulierung des Glücksspielsektors deutlich gemacht. Selbst auf der Ebene der Europäischen Kommission gelang es dem für den Binnenmarkt zuständigen Kommissar McCreevy seither nicht, zumindest eine Mehrheit für die Durchführung der gegen zahlreiche Mitgliedstaaten im Vorstadium anhängigen 
Vertragsverletzungsverfahren wegen der behaupteten Verletzung der BinnenmarktPrinzipien durch die rechtliche und faktische Ausgestaltung ihrer nationalen Glücksspielmärkte zu organisieren.

Allerdings ist die anstehende Öffnung des französischen Markts für OnlineGlücksspiele ein deutliches Indiz für die sich verbreitende Erkenntnis, dass zumindest der Markt für Online-Glücksspiele durch Verbote und klassischen Monopolschutz auf nationaler Ebene allein nicht kontrolliert werden kann und die nominelle Aufrechterhaltung eines nationalen Monopols leicht mit der weitgehenden Verlagerung des Glücksspiels auf ausländische Online-Anbieter bezahlt werden muss. Ein vom französischen Ratsvorsitz im November 2008 vorgelegter Bericht über den Regulierungsstand in den Mitgliedstaaten mit der ergebnisoffenen Frage nach dem Regulierungsbedarf auf EU-Ebene betonte daher mit gutem Grund die Notwendigkeit von EU-Maßnahmen auf diesem Gebiet. Eine Koordinierung der mitgliedstaatlichen Aufsichtsbehörden gegenüber (aus Verbraucherstaatssicht) ,,illegalen“ Anbietern findet bisher nicht statt, wobei die Tätigkeit eines in einem anderen Mitgliedstaat rechtmäßig operierenden Anbieters allenfalls auf dem Gebiet des abgeschotteten Wohnsitzland des Verbrauchers ,illegal“" sein kann; die bloße Teilnahme des Verbrauchers aus einem solchen Land an in einem anderen Mitgliedstaat zugelassenen Glücksspielen darf aufgrund der auch vom Verbraucher in Anspruch zu nehmenden Dienstleistungsfreiheit aus Gründen des europäischen Primärrechts sicher nicht verboten werden. Dabei muss man sich bei Ländern mit staatlich garantierten Monopolen die prinzipiell unauflösliche, in einigen Fällen sogar personifizierte staatliche Interessenskollision deutlich machen, wie sie im Urteil des Bundesverfassungsgerichts vom 28. Juni 2006 so schön zum Ausdruck kommt: Während der Staat als Verbraucherschützer und Spielsuchtbekämpfer auf eine Eindämmung des Glücksspiels hinwirken muss, hat er als Empfänger der Monopolabgaben ein manifestes Interesse an der Aufrechterhaltung und sogar Ausweitung eines hohen Glücksspielumsatzes.

Vor diesem Hintergrund organisierte die ERA im Juni 2009 in enger Zusammenarbeit mit dem tschechischen Ratsvorsitz in Prag eine Tagung über das „Glücksspiel im Binnenmarkt“, deren Ziel die Klärung sachlicher Vorfragen (EU-vertragsrechtliche Grenzen für nationale Zugangsbeschränkungen; die Effizienz unterschiedlicher nationaler Regelungsmodelle bei der Suchtbekämpfung; Existenz eines spezifischen Kriminalitätsrisikos beim Online-Glücksspiel; die Möglichkeit der GlücksspielBesteuerung im Bestimmungsland) und die Eröffnung eines offenen Meinungsaustauschs über die Notwendigkeit und eventuell den Inhalt einer gemeinschaftlichen Regelung sein sollte. ${ }^{1}$ Erfreulicherweise waren alle beteiligten Spieler (Regulatoren und Aufsichtsbehörden, Monopolanbieter und freie Wettbewerber, Verbraucher) bei der Tagung vertreten.

Die auf der Tagung erstatteten Beiträge brachten wichtige Klärungen für manche der Debatte um das am besten geeignete Regulierungsmodell zugrunde liegenden Fragen. So machte das (in diesem Heft nicht abgedruckte) Referat von Professor Gerhard Meyer von der Universität Bremen zur Effizienz unterschiedlicher Regelungsmodelle bei der Suchtbekämpfung deutlich, dass ein strenges Monopolsystem

\footnotetext{
${ }^{1}$ Die Debatte sollte darüber hinaus die Sitzung einer am Folgetag unter tschechischem Vorsitz zusammentretenden Ratsarbeitsgruppe mit Schwerpunkt „Online-Gambling“ vorbereiten.
} 
zwar unter Idealbedingungen die beste Gewähr für den Spielerschutz bieten mag, doch ließen die von ihm vorgelegten Statistiken aus der realen Glücksspielwelt keinen Rückschluss auf eine direkte Korrelation zwischen Regelungssystem und Suchtprävalenz zu. So zeigten sich für Deutschland und das Vereinigte Königreich mit sehr unterschiedlichen Marktregelungen ähnlich hohe Prävalenzraten, für Schweden und Finnland mit strengen Monopolregimen dagegen sogar doppelt so hohe Prävalenzraten wie für das Vereinigte Königreich. - Zum immer wieder ins Feld geführten besonderen Kriminalitätsrisiko arbeitete der unten abgedruckte Beitrag von Michael Levi heraus, dass es keinen Beleg für ein erhöhtes spezifisches Kriminalitätsrisiko im Bereich des Online-Gambling gebe; für Geldwäsche-Zwecke sei E-Gambling sogar besonders ungeeignet, weil Bareinnahmen aus Verbrechen gerade nicht unmittelbar eingesetzt werden könnten und Online-Gambling für unbare Verbrechensprofite keine leichteren Geldwäsche-Optionen biete als andere Verfahren. Nach seiner Auffassung böten dagegen Regulierung und Kontrolle der Anbieter in einem offenen Markt bessere Kontrollmöglichkeiten als eine Prohibitionslösung, die Anbieter in die Illegalität dränge.

Der nachvollziehbaren, wenn auch keinen Eingriff in die Dienstleistungsfreiheit rechtfertigenden Besorgnis der Mitgliedstaaten, eine Marktöffnung für ausländische Anbieter könne leicht zu einem erheblichen fiskalischen Ausfall führen, begegnet der Beitrag von René van der Paardt. Ihm gelingt es aufzuzeigen, dass nach dem Modell der ab 2010 bei „B2C“'-Lieferungen geltenden Umsatzsteuererhebung im Bestimmungsland auch eine Ausgestaltung der Glücksspielsteuer-Erhebung als Verbrauchssteuer im Bestimmungsland konzeptionell möglich und umsetzbar wäre. ${ }^{2}$

Mit den speziellen Problematiken der deutschen Regulierung des Glücksspielmarkts befasst sich der Beitrag von Christian Koenig, der zur Rechtfertigung einer Marktabschließung strenge gemeinschaftsrechtliche Kohärenzanforderungen an die nationale Regelung des Glücksspielmarkts stellt. Diese Kohärenzanforderungen sieht er für Deutschland nicht als gegeben an, weil hier die besonders suchtfördernden Automatenspiele durch freie Lizenzvergabe nach der Gewerbeordnung liberalisiert sind und Spiele mit eher geringem Suchtpotential wie Lotterien oder Sportwetten nur durch Staatsmonopole angeboten werden dürfen. - Während Deutschland mit dem zunächst auf vier Jahre Laufzeit angelegten Glücksspielstaatsvertrag der Länder den Status quo zu zementieren versucht, lässt sich das bisher streng monopolistisch ausgerichtete Frankreich auf dem Gebiet des Online-Glücksspiels mit dem Projekt der Loi Woerth auf eine vorsichtige Öffnung und Liberalisierung ein. Der designierte Chef der künftigen Regulierungsbehörde für Online-Spiele ARJEL François Vilotte stellt den wesentlichen Regelungsgehalt des von der Nationalversammlung in erster Lesung am 13. Oktober 2009 verabschiedeten und derzeit dem Senat vorliegenden Gesetzentwurfs vor, der ein unbeschränktes Lizenz-System für Online-Sportwetten, Online-Pferdewetten und Online-Poker vorsieht, den Marktzugang allerdings auf in Frankreich lizenzierte Anbieter beschränkt. Noch ist offen, ob und mit welchem Inhalt der Entwurf endgültig verabschiedet wird; der ursprünglich ins Auge gefasste

\footnotetext{
${ }^{2}$ Auf der Veranstaltung vertrat auch der österreichische Berichterstatter Franz-Philipp Sutter die Auffassung, die Ausgestaltung der Glücksspielsteuern als Verbrauchssteuern sei geeignet zu gewährleisten, dass diese Abgaben im Bestimmungsland verblieben.
} 
Zeitpunkt des Inkrafttretens vor der Fußballweltmeisterschaft 2010 wird allerdings kaum mehr zu halten sein.

Im Zeitpunkt der unseren Beiträgen zugrunde liegenden Prager Veranstaltung lag die Vorabentscheidung des Europäischen Gerichtshofs in der Sache Liga Portuguesa ./. Santa Casa da Misericórdia vom 8. September $2009^{3}$ noch nicht vor, die von den Verteidigern der Staatsmonopole nicht ganz grundlos als Etappensieg gefeiert worden ist. Zwar nimmt der EuGH in diesem Urteil grundsätzlich auf seine frühere Rechtsprechung zur Rechtfertigung grundfreiheitsbeschränkender nationaler Maßnahmen Bezug. Erstaunlich ist jedoch, wie leicht der Gerichtshof den Staaten nunmehr die Berufung auf einen anerkannten ,zwingenden Grund des Allgemeininteresses“ (hier der Kriminalitätsbekämpfung) zur Rechtfertigung des festgestellten Eingriffs in die Dienstleistungsfreiheit macht, denn offensichtlich erspart nach der schon im Apotheken-Urteil ${ }^{4}$ erkennbaren Linie dieser Rechtsprechung die bloße Behauptung einer spezifischen Gefahr deren konkrete Darlegung, so dass es auf eine Beweisführung oder zumindest eine Plausibilitätsdarlegung ebenso wenig ankommt wie auf den Nachweis von Erforderlichkeit und Verhältnismäßigkeit der ergriffenen Eingriffsmaßnahme: Der geltend gemachte ,mögliche“ oder ,,eventuelle“ Betrug „,durch die Anbieter" (also die in anderen Mitgliedstaaten zugelassenen Anbieter) wird von der portugiesischen Regierung nur behauptet, nicht belegt, obwohl doch gerade die Wettskandale der jüngsten Zeit Monopolmärkte nicht weniger als liberalisierte Märkte betrafen.

Vor dem Gerichtshof sind zahlreiche weitere Vorabentscheidungen insbesondere aus Deutschland anhängig, die auch eine Stellungnahme des Gerichtshofs zum von Koenig festgestellten Kohärenzdefizit der deutschen Glücksspielregulierung erwarten lassen. ${ }^{5}$ Die immer umfangreichere, den Besonderheiten der vorgelegten Fälle verhaftete Kasuistik des Gerichtshofs lässt inzwischen im Interesse einer größeren Rechtssicherheit allerdings selbst Gegner einer europäischen Harmonisierung nach einer europäischen Rahmenregelung rufen, die die Kompetenz der Mitgliedstaaten zur Festlegung ihres eigenen Regelungsmodells unterstreichen, im grenzüberschreitenden Bereich Rechtssicherheit schaffen, illegale Anbieter aus OffshorePlätzen vom Markt fernhalten, die Einnahmen für „good causes“ sichern und die mitgliedstaatlichen Maßnahmen zur Bekämpfung illegaler Glücksspielaktivitäten koordinieren soll. ${ }^{6}$ Ob eher offensiv den Marktfreiheiten oder defensiv den zugangsbeschränkenden Partikularregelungen zugewandt, das Publikum erwartet die Initiativen der neuen Kommission auf dem Gebiet des Glücksspiels mit besonderer Spannung.

\footnotetext{
${ }^{3}$ Rs. C-42/07, Urteil der Großen Kammer des Gerichtshofs vom 8.9.2009 (noch nicht in der amtl. Slg. veröffentlicht).

${ }^{4}$ Verb. Rs. C-171/07 und 172/07, Urteil vom 19.5.2009 (noch nicht in der amtl. Slg. veröffentlicht).

${ }^{5}$ Koenig weist zu Recht darauf hin, dass die deutsche Monopolregelung in erster Linie auf die Suchtbekämpfung abstellt, und warnt daher vor einer Übertragung der Grundsätze der Liga-PortuguesaEntscheidung (in EuZW 19/2009, 673). Der Schlussantrag von Generalanwalt Yves Bot vom 26.1.2010 in der Rs. C-409/06 Winner Wetten, betont gleichfalls die Notwendigkeit einer kohärenten Regelung und könnte enorme Sprengkraft für die deutsche Regulierung des Glücksspielmarktes entfalten.

${ }^{6}$ Diese Position wurde beispielsweise von Rechtsanwalt Philippe Vlaemminck, Gent, auf der Schlussdiskussion der Prager ERA-Tagung vertreten.
} 